Revista de Derecho

\title{
Violencia contra las mujeres y justicia de las mujeres en la Amazonía
}

Violence against women and justice from women in the Amazon

\section{Milena Justo Nieto}

Abogada, Ecuador

mjustonieto@gmail.com

ORCID: 0000-0002-7821-3810

DOI: https://doi.org/10.32719/26312484.2020.34.5

Fecha de recepción: 25 de octubre de 2019

Fecha de aceptación: 7 de enero de 2020 


\section{RESUMEN}

El presente trabajo analiza las nuevas formas de violencia contra las mujeres que se desatan en la Amazonía, en un contexto de extractivismo y explotación. Mediante fuentes secundarias, se estudia la relación entre la precarización de la vida y el recrudecimiento de las formas de violencia contra las mujeres y contra todo lo feminizado: el cuerpo de las mujeres, la naturaleza, la cultura. Una de las formas identificadas de violencia que se desata en la Amazonía es la violencia institucional contra las mujeres, referida a la violencia del sistema de justicia y del Estado cuando este es permisible ante la impunidad e indiferencia frente a los casos suscitados en esta zona, que acarrean mayores barreras de acceso a justicia. A partir de ello, se introduce una propuesta teórica en relación a las respuestas de las mujeres frente a la impunidad de los casos de violencia en su contra y la precariedad de la vida, la cual se fundamenta en el pluralismo jurídico emancipador. Se postula el desarrollo de una agencia de las mujeres frente a la crisis, manifestaciones de resiliencia que pueden convertirse en formas de hacer justicia desde las prácticas sociales, en este caso desde las prácticas de las mujeres en la Amazonía.

PALABRAS CLAVE: mujeres, Amazonía, violencia, pluralismo jurídico emancipador.

\section{ABSTRACT}

This his paper analyzes the new forms of violence against women that are unleashed in the Amazon, in a context of extractivism and exploitation. Through secondary sources, the relationship between the precariousness of life and the resurgence of forms of violence against women and against everything feminized is studied: the body of women, nature, culture. One of the forms of violence that is identified is unleashed in the Amazon, is the institutional violence against women, referred to the violence of the justice system and the State when it is permissible in the face of impunity and indifference to the cases raised in this area, which carry greater barriers to access justice. From this, a theoretical proposal is introduced in relation to women's responses to the impunity of cases of violence against them and the precariousness of life, which is based on emancipatory legal pluralism. The development of an agency of women in the face of the crisis is postulated, manifestations of resilience that can become ways of doing justice from social practices, in this case from the practices of women in the Amazon.

KEYWORDs: women, Amazon, violence, emancipatory legal pluralism. 


\section{NUEVAS FORMAS DE VIOLENCIA CONTRA LAS MUJERES EN LA AMAZONÍA}

$\mathrm{L}$ os actuales índices de violencia contra las mujeres ${ }^{1}$ y las formas en que se propaga, ${ }^{2}$ demandan nuevas miradas, nuevas nociones y particulares condiciones de análisis del aquí y ahora. Solo en las 4 últimas décadas se ha devastado gran parte de la Amazonía. Según el Organismo Supervisor de la Inversión en Energía y Minería (Osinergmin), entre 1997 y julio de 2016 se produjeron 190 derrames de petróleo registrados oficialmente por el Estado peruano, pero los pobladores han detectado muchos más. ${ }^{3}$ Frederica Barclay relaciona el período cauchero con el actual período petrolero; ambos tienen puntos en común: el carácter extractivo, la subordinación de las economías locales, el consumo voraz de recursos. ${ }^{4}$ Marc Gavaldá identifica además que en la década de 1970 en la Amazonía peruana las concesiones petroleras abrieron las puertas del mercado a las comunidades; ello costó que actividades como la caza, pesca, agricultura y recolección fueran sustituidas por la compra de comestibles, dada la contaminación de sus recursos. La modernización también aceleró la erosión cultural con la incursión de instituciones como escuelas, religiones, televisión. ${ }^{5}$

Con base en lo señalado en el párrafo anterior, los impactos de la industria petrolera, el extractivismo y/o las llamadas actividades de desarrollo son visibles en los ríos, flora y fauna de la Amazonía, ${ }^{6}$ pero también en las relaciones sociales, sistemas

1. Según el Ministerio de la Mujer y Poblaciones Vulnerables (MIMP), en el año 2019 en el Perú se han registrado 168 feminicidios, la cifra más alta de la última década. Las cifras oficiales de la Policía Nacional del Perú (PNP) por denuncias recibidas en las Comisarías en 2018 alcanzaron 222.376 denuncias por "violencia familiar", y 7.789 denuncias por violación sexual.

2. El Plan Nacional Contra la Violencia de Género 2016-2021 (D.S. n. ${ }^{\circ}$ 008-2016-MIMP) reconoce 4 tipos de violencia y 16 modalidades de violencia de género, entre estas últimas se encuentra la violencia en conflictos sociales, aunque a la actualidad no se realice una medición o seguimiento de esta modalidad de violencia, su reconocimiento evidencia la preocupación por su incidencia en algunos sectores del país donde las mujeres se encuentran en mayor riesgo de ser víctimas de violencia de género en el contexto de conflictos sociales.

3. Yvette Sierra Praeli, “¿Qué ha pasado en 40 años de actividad petrolera en la Amazonía peruana?”, $M O N-$ GOBAY: Periodismo ambiental independiente, 23 de junio de 2017, https://es.mongabay.com/2017/06/ ha-pasado-40-anos-actividad-petrolera-la-amazonia-peruana/.

4. Frederica Barclayro, “¿Qué ha significado el petróleo en la configuración de Loreto como una región?, en Foro 40 años de explotación petrolera en Loreto. Lecciones aprendidas", SPDA Actualidad Ambiental, 23 de noviembre de 2011, http://www.actualidadambiental.pe/wp-content/uploads/2011/11/Qu\%C3\%A9ha-significado-el-petr $\% \mathrm{C} 3 \% \mathrm{~B} 31$ eo-en-la-consolidaci $\% \mathrm{C} 3 \% \mathrm{~B} 3 n$-de-Loreto-como-una-regi $\% \mathrm{C} 3 \% \mathrm{~B} 3 \mathrm{n}$ Frederica-Barclay.pdf.

5. Marc Gavaldá, Gas amazónico: los pueblos indígenas frente al avance de las fronteras extractivas en Perú, 1a. ed., Akadēmeia; Ecología humana 142 (Barcelona: Icaria, 2013), 42.

6. Se hace mención al impacto del extractivismo tanto de la industria minera como de hidrocarburos: petróleo, pues en la Amazonía peruana se tiene presencia de actividades de ambos tipos. La Amazonía repre- 
de valores, organización social y relaciones de intercambio con el mundo natural. En los territorios con grandes recursos naturales, como la Amazonía, las relaciones desiguales de género se han instaurado a partir de la modernidad/colonialidad y la hetero-normatividad naturaleza/cultura, hombre/mujer, ${ }^{7}$ generando que la violencia contra las mujeres se agudice, como lo demuestran investigaciones realizadas sobre territorios rurales con presencia de comunidades. ${ }^{8}$ En Esmeraldas, Ecuador, por ejemplo, Michel Lapierre y Aguasantas Macías refieren que "el patriarcado, presente bajo ciertas formas en las sociedades ancestrales, se exacerba con el modelo de sociedad que existe en Esmeraldas, construido de forma protagónica en las últimas décadas por las actividades extractivas, el estado y crimen organizado"; 9 la actividad extractiva ha resquebrajado las relaciones de género, principalmente de las mujeres indígenas y afrodescendientes. En ese marco, el extractivismo ha establecido un nuevo orden sobre los territorios que explota, en los cuales se desarrollan nuevas formas de violencia contra las mujeres.

Para comprender estas formas de violencia se usará la teoría desarrollada por Rita Segato sobre las nuevas formas de violencia contra las mujeres, aplicándola al contexto social, geográfico y político de la Amazonía peruana. Para la autora, "es necesario introducir en la retórica jurídica y en la consciencia de la opinión pública la centralidad y el significado de las nuevas formas de victimización del cuerpo femenino en las estrategias de manutención de un orden basado en la dominación arbitraria y soberana

senta el mayor porcentaje del territorio peruano (61\%), pero su densidad poblacional es baja (Instituto de Investigaciones de la Amazonía Peruana, Delimitación del territorio amazónico con criterio ecológico y criterio hidrográfico. Lima: IIAP, 1998). Javier Pulgar Vidal dividió este territorio por sus pisos altitudinales, diferenciados en dos: selva alta y selva baja (Javier Pulgar Vidal, Las ocho regiones naturales del Perú. Lima: Ausonia, 1963). La selva alta está integrada por departamentos como Madre de Dios y Amazonas, en los que la principal actividad extractiva es la minería. La selva baja está integrada por departamentos como Loreto (que ocupa la mayor parte del territorio amazónico), donde la principal actividad extractiva es el petróleo. No obstante, algunos estudios dan cuenta de pequeñas concesiones mineras y minería ilegal en las cuencas amazónicas de Loreto (Inversión para el desarrollo e integración regional, Encuentro económico de la región Loreto. Iquitos: Gobierno regional de Loreto, octubre de 2009). La propuesta del presente artículo es una aproximación teórica, por lo que no se hablará de un territorio específico de la Amazonía y los pueblos indígenas que la integran, sino de la Amazonía en su totalidad y generalidad, pues la intención es el acercamiento teórico y no el estudio de caso.

7. Astrid Ulloa, "Feminismos territoriales en América Latina: defensas de la vida frente a los extractivismos" NÓMADAS 45 | octubre de 2016 - Universidad Central - Colombia, NÓMADAS, n. ${ }^{\circ} 45$ (octubre de 2016): 126, http://nomadas.ucentral.edu.co/index.php/component/content/article?id=887.

8. Ibíd.

9. Michel Lapierre Robles y Aguasantas Macías Marín, Extractivismo, (neo)colonialismo y crimen organizado en el norte de Esmeraldas (Quito: Abya-Yala / Pontificia Universidad Católica del Ecuador / Instituto de Estudios Ecologistas del Tercer Mundo, 2018), 275. 
sobre la vida de las personas y sus territorios". ${ }^{10}$ La autora, tomando el paradigma del biopoder, explica cómo la red de los cuerpos pasa a ser el territorio, en relación a los intereses del gobierno de turno. ${ }^{11}$ Segato indica, además, que estas nuevas formas de violencia constituyen una guerra configurada por un universo para-estatal de control, que captura la vida social y la política como vehículos de una violencia expresiva: una violencia que transmite un mensaje de impunidad. Todo esto se suscita en el marco del segundo estado, el cual es viable por la permisibilidad y silencio del Estado nacional.

El contexto de violencia al que hace referencia Segato se basa en lo que observó en Ciudad Juárez; en este escenario de crímenes impunes contra las mujeres, las madres de las víctimas pedían que se ajusticie a los reales responsables de los feminicidios, que representaban a poderes mayores. ${ }^{12}$ Un escenario de crueldad e impunidad semejante podría extrapolarse a lo que ocurre en la Amazonía peruana, en un departamento como Madre de Dios, donde la minería ilegal ha desencadenado la trata de personas con fines de explotación sexual, el crimen organizado, entre otros crímenes que permanecen impunes por el gran poder de la minería ilegal y las mafias alrededor de ella. ${ }^{13}$ No obstante, en otros territorios de la Amazonía, el petróleo, la minería, la maderería y otras actividades de desarrollo, al generar de modo colateral la contaminación de los ríos y la naturaleza, han generado a su vez la precariedad de la vida, lo que ha alterado notablemente la dinámica social de las comunidades, trayendo escasez y diferentes formas de violencia. ${ }^{14}$

Vandana Shiva sostiene que existe una conexión entre la intensificación de la violencia contra las mujeres y el aumento de las políticas económicas neoliberales, no como origen, mas sí en la adopción de nuevas formas de violencia. ${ }^{15}$ La violencia contra las mujeres y la naturaleza no está determinada por los genes, sino por un pa-

10. Rita Laura Segato, Las nuevas formas de la guerra y el cuerpo de las mujeres (Buenos Aires: Tinta Limón, 2013), 71.

11. Ibíd., 33.

12. Rita Segato, La violencia contra las mujeres es sobre las mujeres contra la vida, entrevistada por Nadia Fink, Fundación Rosa Luxemburgo, 7 de agosto de 2018, http://www.biodiversidadla.org/Documentos/ La_violencia_contra_las_mujeres_es_sobre_las_mujeres_contra_la_vida._Dialogo_con_Rita_Segato.

13. Jaris Mujica, Elementos comparados del impacto de la trata de personas en la salud de víctimas adolescentes en el contexto de la minería ilegal de oro en Madre de Dios (Lima: OMS-OPS / Anesvad / PROMSEX Centro de Promoción y Defensa de los Derechos Sexuales y Reproductivos, 2014).

14. Ver Marc Gavaldá, El avance de la frontera petrolera amenaza a los pueblos en aislamiento voluntario (Servindi, 2012); Rosario Hurtado, "Río de petróleo en Loreto", Ideele Revista, n. . 246; Claudia Grados y Eduardo Pacheco, "El impacto de la actividad extractiva petrolera en el acceso al agua: el caso de dos comunidades kukama kukamiria de la cuenca del Marañón (Loreto, Perú)”, Anthropológica XXXIV, n. ${ }^{\circ}$ 37 (2016): 33-59, entre otros.

15. María Mies y Vandana Shiva, Ecofeminismo: teoría, crítica y perspectivas (Barcelona: Icaria, 2014 ), 19. 
radigma social cuyo nombre es patriarcado capitalista. ${ }^{16}$ En ese sentido, los territorios y cuerpos amenazados que se explotan y violentan en la Amazonía, el cuerpo de las mujeres y la misma naturaleza, alcanzan niveles cada vez mayores en el marco del modelo capitalista que caracteriza a la industria extractiva.

Las formas de violencia contra las mujeres desatadas en contextos como el de la Amazonía se cometen contra todo lo feminizado: la naturaleza y los cuerpos femeninos, lo comunitario y lo popular. Raquel Gutiérrez y Dawn Paley, al referirse a la violencia y asesinato de mujeres, comentan que esta violencia es también en contra de las formas de vida y los entramados comunitarios que resisten y crean en medio del huracán liberal de despojo, súper-explotación y muerte. ${ }^{17}$ Estas formas de violencia muchas veces no son visibles en las cifras oficiales o problemáticas nacionales, pero pueden identificarse desde el análisis teórico del contexto particular de la Amazonía y sus dinámicas, centrándonos en la Amazonía amenazada por la industria extractiva.

\section{VIOLENCIA CONTRA LAS MUJERES EN CONTEXTOS EXTRACTIVOS}

Las actividades extractivas legales e ilegales en territorios rurales son posibles por el Estado y las soberanías superpuestas que manejan el control económico, y por tanto territorial; este control se vuelve desmedido por el poder que despliegan las actividades extractivas. Astrid Ulloa, en un estudio de territorios afectados por actividades extractivas, señala cómo se van arrogando poco a poco, en estos territorios, licencias que anulan la voluntad del otro, por ejemplo, la imposición y el desinterés por el consentimiento previo, libre e informado de la población a partir del cual aparecen las demandas indígenas en contra de la apropiación de sus territorios..$^{18}$ Esto ocurre porque los gobiernos, como el de Perú, promueven políticas extractivistas como vías de acceso para el cumplimiento de metas de desarrollo nacionales. ${ }^{19}$ Estos proyectos extractivos por tanto se relacionan con el desarrollo, el cual es relativo, pues principalmente es construido desde características del ser androcéntrico occidental y desde una mirada masculina no dialogada, cuyos beneficios no son para las mujeres, ni pueblos indígenas, como bien se ha identificado a través de los procesos de descolonización

16. Ibíd., 34.

17. Raquel Gutiérrez y Dawn Paley, "La transformación sustancial de la guerra y la violencia contra las mujeres en México", Deportate, esuli, profughe DEP, Rivista telemativa di studi sulla memoria femminile 30 (2016): 4, https://www.unive.it/media/allegato/dep/n30-2016/01_Aguillar-Dawn-Palley.pdf.

18. Ulloa, "Feminismos territoriales en América Latina: defensas de la vida frente a los extractivismos".

19. Denise Bebbington et al., Evaluación y alcance de la industria extractiva y la infraestructura en relación con la deforestación: Amazonía (Lima: Derecho, Ambiente y Recursos Naturales (DAR), 2019), 10. 
y despatriarcalización de diversos feminismos del sur. ${ }^{20}$ Rocío Silva va a decir que cuando hablamos de extractivismo nos encontramos con una confrontación entre dos maneras de ver el mundo con propuestas irreconciliables, para lo cual propone entender sus alcances e impactos como un proyecto biopolítico. ${ }^{21}$

En el estudio realizado por Ulloa, del análisis de los impactos de las actividades extractivas en América Latina, se identifica que los proyectos extractivos agudizan las desigualdades e incrementan la violencia contra las mujeres, pero también afectan los modos de vida tanto de hombres como de mujeres. ${ }^{22}$ Silva observa cómo desde la colonialidad del poder se han desarrollado formas de dominación que han instaurado la inferioridad racial de los indígenas, lo que ha significado su subalternización y una suerte de feminización del indígena para justificar su dominación. ${ }^{23}$ Ello se enlaza con la idea de la violencia contra todo lo feminizado: la naturaleza, la cultura, la comunidad y el cuerpo de las mujeres. Es cierto que esta inferiorización del indígena puede ser entendida como feminización; no obstante, dentro de este grupo hay un grupo más subalternizado, que son las mujeres indígenas.

Los impactos del extractivismo sobre el cuerpo de las mujeres son mayores, como lo señalan diversas autoras desde el estudio de contextos extractivos en distintos puntos de América Latina. ${ }^{24}$ Entre los impactos diferenciados sobre las mujeres que identifica Silva, menciona la afectación a la soberanía alimentaria por la dificultad para acceder a los alimentos (por contaminación de suelos, desvío de ríos, etc.), ${ }^{25}$ esto afecta más a las mujeres porque son ellas las encargadas de recolectar, distribuir y preparar los alimentos, así como de enfrentar un conflicto familiar por no proveerlo. Silva también menciona testimonios de la audiencia realizada por la Comisión Interamericana

20. Miriam Lang et al., "Pensar desde el feminismo: Críticas y alternativas al desarrollo", en Más allá del desarrollo, ed. Grupo Permanente de Trabajo sobre Alternativas al Desarrollo (Quito: Fundación Rosa Luxemburg / Abya-Yala, 2011).

21. Rocío Silva Santisteban, Mujeres y conflictos ecoterritoriales: impactos, estrategias, resistencias (Lima: Centro de la Mujer Peruana Flora Tristán / DEMUS Estudio para la Defensa de los Derechos de la Mujer / Coordinadora Nacional de Derechos Humanos / AIETI Asociación de Investigación y Especialización sobre Temas Iberoamericanos / Entre pueblos, 2017), 17-9.

22. Ibíd., 124.

23. Silva Santisteban, Mujeres y conflictos ecoterritoriales, 70.

24. Ver Bolados García y Sánchez Cuevas, "Una ecología política feminista en construcción: El caso de las 'Mujeres de zonas de sacrificio en resistencia', Chile", Psicoperspectivas, n. ${ }^{\circ} 16$ (2017): 33-42, https://www. psicoperspectivas.cl/index.php/psicoperspectivas/article/viewFile/977/659; Camila Rolando Mazzuca et al., "Violencia contra mujeres tejedoras de resistencias", Ecología Política, n. 53 (2017): 104-7, https://www. jstor.org/stable/26333549; Ulloa, "Feminismos territoriales en América Latina: defensas de la vida frente a los extractivismos"; Silva Santisteban, Mujeres y conflictos ecoterritoriales.

25. Silva Santisteban, ibíd., 33. 
de Derechos Humanos (CIDH) en Panamá, ${ }^{26}$ como el de Jacqueline Vinari Pangoa, lideresa machiguenga asháninca, quien denuncia como principal problemática para las mujeres: la seguridad alimentaria y las enfermedades que estas acarrean para ellas y los niños. ${ }^{27}$ En esa misma audiencia, la dirigente kukama Gladys Dávila coincide en que es una de las principales afectaciones: "las mujeres viven abandonadas, solas, y los niños tienen enfermedades extrañas [...]. Nosotras como mujeres curamos a nuestros niños, pero ahora no lo podemos conseguir. Es causa de la empresa petrolera. No podemos producir porque los suelos están contaminados, queremos hacer nuestras siembras de verduras, pero no podemos". ${ }^{28}$

Por otro lado, la afectación a la soberanía alimentaria no impacta solo sobre el suelo de la comunidad donde se ubica la industria extractiva, sino a su alrededor: en la Amazonía un derrame en la comunidad donde se ubique el pozo petrolero puede contaminar toda la cuenca. La incapacidad de obtener alimento, animales, peces, agua de su propio territorio, como es lo habitual desde las actividades primarias en comunidades, desencadena la precarización de las familias. ${ }^{29}$ Uno de los desenlaces es el desplazamiento, la migración, pues, con tierras contaminadas y/o expropiadas, las familias se mudan a las ciudades y en esos espacios la situación empeora. Silva menciona una entrevista a Raquel Coca, quien relata los temores y peligros ante la migración: "La posibilidad de dejar sus territorios para asimilarse al ámbito urbano es difícil no solo porque tendrían que cambiar su modo de vida practicada muchos años, sino que también tendrían que insertarse laboralmente, situación sumamente complicada debido a que las mujeres que viven en zonas rurales siguen siendo el grupo poblacional analfabeto más grande del país". ${ }^{30}$ Entre otros aspectos que resalta la autora está la dinámica entre el capitalismo por despojo y la masculinización de la propiedad de la tierra como principales factores que producen la vulnerabilidad en las mujeres. ${ }^{31}$

26. Organización de Estados Americanos, Audiencia sobre denuncias de violaciones de derechos humanos de las mujeres en el contexto de actividades extractivas en Perú, 159 Período de Sesiones, Panamá, 2 de diciembre de 2016.

27. Ibíd., 39.

28. Ibíd., 40-1.

29. Como da cuenta lo ocurrido en la Comunidad de Cuninico, en la que se registró un derrame de petróleo de 2.358 barriles del Oleoducto Norperuano en junio de 2014, y en la cual hasta 2 años después se encontró presencia de metales pesados con valores por encima de lo permitido en la mitad de la población. La contaminación del río significó una afectación a la vida e integridad personal de los pobladores de las comunidades aledañas, quienes hasta 3 años después no podían acceder a las aguas de su río, ni a la caza y pesca, debiendo recurrir a la compra de alimentos y agua, razón por la cual en el año 2017, la CIDH emite la Resolución 52/2017 de medidas cautelares a favor de los pobladores de las comunidades.

30. Ibíd., 33.

31. Silva Santisteban, Mujeres y conflictos ecoterritoriales, 34. 
La violencia en estos contextos se da de diversas formas: antes, durante o después de la actividad extractiva. Pese a que en el estudio anteriormente citado la autora analiza principalmente la violencia durante las movilizaciones o crisis de los conflictos sociales, ${ }^{32}$ se pueden producir otras violencias contra las mujeres directas e indirectas, tanto contra las que están dentro del territorio en disputa como contra las que no lo están. Por ejemplo, la violencia en relación de pareja y violencia sexual puede ir en aumento, se pueden presentar nuevas enfermedades que atacan a la población, la inseguridad en consecuencia se agudiza y se vuelve un factor de peligro: todo en un marco de precarización (contaminación de los suelos, dificultades para conseguir alimentos, entre otros) que causa mayores conflictos a la comunidad y a las mujeres.

La situación empeora cuando, frente a estas violencias e impactos evidentemente negativos sobre las mujeres, se responde con impunidad. Estos efectos son tan evidentes que, en un informe de la CIDH, se alerta que "La violencia contra las mujeres indígenas está presente durante la ejecución de importantes proyectos de desarrollo, inversión y extracción [...]. La respuesta de las autoridades estatales a la mayoría de estos actos es nula o deficiente, por lo que permanecen en la impunidad". ${ }^{33}$ Del mismo modo, la Recomendación $\mathrm{n}^{\circ} 30$ de la $\mathrm{Cedaw}^{34}$ sobre las mujeres en la prevención de conflictos y en situaciones de conflicto señala: "Los conflictos agravan las desigualdades existentes entre los géneros y el riesgo de las mujeres de ser víctimas de distintas formas de violencia de género por agentes estatales y no estatales". Se debe considerar que los conflictos sociales afectan tanto a la comunidad donde se desarrolla el conflicto como a las comunidades de las zonas cercanas y lejanas.

Los contextos extractivos recrudecen la violencia contra las mujeres. Sumando a lo anteriormente mencionado, Ulloa presenta el caso de Chocó, Colombia, lugar donde la consolidación de la minería ilegal significó el auge de espacios comerciales aledaños a los complejos de extracción, en los cuales se desarrolla la trata con fines de explotación sexual contra mujeres y niñas, ${ }^{35}$ situación semejante a la ya referida en Madre de Dios, Perú. Ambos casos encarnan la forma en cómo los cuerpos-territorios de las mujeres son apropiados mediante despojos cotidianos y expropiaciones de modos de vida. Se reitera: los efectos de esta violencia no son solo sobre los territorios -na-

32. Ibíd., 86 .

33. Organización de los Estados Americanos y Comisión Interamericana de Derechos Humanos, "Las mujeres indígenas y sus derechos humanos en las Américas" (Organización de los Estados Americanos, 2017), 12.

34. Organización de Organización de Naciones Unidas, Comité para la eliminación de todas las formas de discriminación contra la mujer, Recomendación general n. ${ }^{\circ} 30$, sobre las mujeres en la prevención de conflictos y en situaciones de conflicto y posteriores a conflictos, $56 .^{\circ}$ período de sesiones, 1 de noviembre de 2013, párrafo 34.

35. Ulloa, "Feminismos territoriales en América Latina: defensas de la vida frente a los extractivismos", 131. 
turaleza y cuerpo de las mujeres- directamente explotados, sino también los aledaños, pues esta situación puede precarizar la vida de las familias, contaminar los ríos, generar mayores enfermedades, alterar las relaciones sociales, poner en peligro el libre tránsito de niñas, niños, adolescentes, incrementar los costes de vida y más. Ninguno de estos casos debe dejarse de denunciar ni atender, por ello es importante la respuesta que da el Estado a través del sistema de justicia formal, los servicios especializados y la atención oportuna y atenta a la población de comunidades, la cual muchas veces no se desarrolla de forma idónea. El nivel de ausencia e impunidad termina generando una nueva forma de violencia contra las mujeres, que es la institucional.

\section{VIOLENCIA INSTITUCIONAL CONTRA LAS MUJERES}

Para Encarna Bodelón, cuando se aborda la violencia contra las mujeres no se visibiliza que la principal razón de la permanencia de los diversos tipos de violencia es la tolerancia e impericia del Estado; para superar esto refiere que se debe salir del androcentrismo jurídico. ${ }^{36}$ Es decir, conocer-ver más allá de las salidas institucionales porque estas expresan la indiferencia y el maltrato ante la violencia contra las mujeres, así como los estereotipos de género dentro del mismo sistema de justicia. Bodelón, como otras autoras, ${ }^{37}$ hace referencia a la lógica sexuada del derecho, falsamente neutral y masculina, que termina fomentando la desigualdad desde las mismas instituciones. La postura de la autora es que el derecho es una herramienta que ha dado su aporte durante mucho tiempo a relaciones sociales patriarcales, pero también es una herramienta que puede ayudar a romper estas relaciones. ${ }^{38}$

Pese a que la violencia contra las mujeres cuenta actualmente con un amplio marco normativo nacional e internacional, y que los instrumentos internacionales suscritos por el país disponen a los Estados actuar con la debida diligencia para prevenir, investigar y sancionar la violencia contra la mujer, ${ }^{39}$ los Estados siguen siendo sancio-

36. Encarna Bodelón, "Violencia institucional y violencia de género", Anales de la Cátedra Francisco Suárez. Revista de Filosofía Jurídica y Política 48 (2014): 132, 137, http://revistaseug.ugr.es/index.php/acfs/ article/view/2783.

37. Ver las autoras: Encarna Bodelón González, "Feminismo y Derecho: Mujeres que van más allá de lo jurídico", en Género y dominación: críticas feministas del derecho y el poder, comp. Gemma Nicolás Lazo et al., 1. ${ }^{a}$ ed. Huellas. Desafío(s) 7 (Rubí, Barcelona: Anthropos, 2009); Alda Facio, "Metodología para el análisis de género del fenómeno legal", y Elisabeth Baninter, "El enigma masculino. La gran X", en El género en el derecho. Ensayos críticos, comp. Judith Salgado, Ramiro Ávila Santa María y Lola Valladares (Quito: Ministerio de Justicia y Derechos Humanos / UNIFEM, 2009), 181-224 y 69-98.

38. Encarna Bodelón González, ibíd., 109.

39. Organización de Estados Americanos, Convención Interamericana para Prevenir, Sancionar y Erradicar la Violencia contra la Mujer (Convención de Belém do Pará), art. 7. 
nados por el incumplimiento de este deber. Gutiérrez y Gagó refieren: "Mientras más se insiste en la 'vigencia del estado de derecho', en la 'observancia de la ley' o en la 'consolidación institucional' del andamiaje burocrático para la administración de la población; más confusa e incomprensible se vuelve la forma mutada de lo bélico". ${ }^{40}$ Del mismo modo, Rita Segato se pregunta cuán eficaces son o conseguirán ser las leyes que criminalizan actitudes fuertemente sustentadas por la moral dominante ${ }^{41}$ Las medidas legislativas no son suficientes para erradicar la violencia contra las mujeres que se suscitan a diario, menos aún si se estacan en el proceso sancionador y desconocen las diferencias que se vive en lo rural y urbano, dejando de lado los procesos participativos y la necesidad de implementar mecanismos de prevención y protección que incluyan el involucramiento de la comunidad.

A ello se le suma la inoperatividad de la justicia formal, que incrementa las barreras ya existentes para el acceso a justicia. Las mujeres indígenas que viven en comunidades de la Amazonía deben atravesar los ríos para acceder a la atención de los operadores de justicia; ello implica navegar durante varios días e invertir un dinero que no se tiene, lo que se agrava cuando la atención desde los operadores de la justicia ordinaria no es la idónea. Diferentes investigaciones dan cuenta de las barreras de acceso a la justicia que enfrentan las mujeres sobrevivientes de hechos de violencia en su contra por un sistema burocrático que no se adecua interculturalmente frente a los casos que se suscitan en comunidades, lo que decae en impunidad. ${ }^{42}$ Con la experiencia de campo y el estudio de la zona rural Javier La Rosa y Wilfredo Ardito identifican que las barreras del acceso a la justicia en el mundo rural con: lingüísticas, económicas, culturales y geográficas. ${ }^{43}$ Ardito analiza tanto aquellas que obedecen a

40. Raquel Gutiérrez y Verónica Gagó, "Prólogo", en Las nuevas formas de la guerra y el cuerpo de las mujeres (Buenos Aires: Tinta Limón, 2013), 10.

41. Rita Laura Segato, "La argamasa jeráquica: Violencia moral, reproducción del mundo y eficacia simbólica del derecho", en Las estructuras elementales de la violencia: Ensayos sobre género entre la antropología, el psicoanálisis y los derechos humanos, 1a. ed., Derechos humanos. Viejos problemas, nuevas miradas (Buenos Aires: Universidad Nacional de Quilmes, Prometeo 3010, 2003), 122.

42. Véase Rocío Franco Valdivia y María Gonzales Luna, Las mujeres en la justicia comunitaria: víctimas, sujetos y actores (Lima: IDL, 2009); Rocío Franco Valdivia, "Miradas cruzadas: el acceso de las mujeres a la justicia desde el género y la cultura", en Acceso a la justicia en el mundo rural, coord. Javier La Rosa Calle (Lima: IDL, 2007); Patricia Balbuena Palacios, Mujeres rurales y justicia de paz (Lima: Justicia Viva, IDL / PUCP, 2005); Patricia Balbuena Palacios, "Acceso a la Justicia con equidad de género: una propuesta desde la Justicia de Paz" (tesis de posgrado, Maestría en Género y Desarrollo, UNSM, 2006); Rocío Villanueva Flores, "Constitucionalismo, pluralismo jurídico y derechos de las mujeres indígenas", Revista de Derecho Público de la Universidad de los Andes, n. ${ }^{\circ} 32$ (junio de 2014); Mercedes Crisóstomo Meza, Violencia contra las mujeres rurales: una etnografia del Estado peruano (Lima: PUCP, Departamento de Ciencias Sociales, 2016).

43. Javier La Rosa Calle, "Acceso a la justicia: elementos para incorporar un enfoque integral de política pública", en Acceso la justicia en el mundo rural, coord. Javier La Rosa Calle (Lima: Instituto de Defensa Legal, 2007), 29-36. 
la estructura de nuestra sociedad como las que se derivan de una serie de decisiones legales o administrativas. ${ }^{44}$ Estas se pueden dividir en: barreras geográficas, de distancia, económicas, y, por otro lado, las barreras lingüísticas y culturales y de funcionamiento del sistema de justicia ordinaria; estas últimas dependen principalmente de las instituciones y sus operadores de justicia.

Con base en las barreras mencionadas en el párrafo anterior, principalmente las barreras de funcionamiento del sistema de justicia, la violencia contra las mujeres en contextos extractivos que se suscita en comunidades de la Amazonía recrudece por los operarios de la costumbre, operadores que no adecuan la atención frente a las diferencias de los casos que provienen de zonas rurales. En ese sentido, las formas de violencias institucionales contra las mujeres que produce el Estado se dan desde muchos frentes: por medio de la indiferencia en la generación de normas que no atienden más allá de lo urbano; la discriminación dentro del sistema de justicia formal para con las autoridades indígenas; las barreras de acceso a justicia económicas, geográficas, de distancia, culturales; y aquellas relacionadas al funcionamiento del sistema de justicia ordinaria; los estereotipos de género, raza/etnia, clase; entre otras. La violencia se ha incrementado y no se cuenta con rutas articuladas de salvaguarda y protección. Este mensaje es doblemente injusto, pues es un mensaje de tolerancia e indiferencia selectiva con la otredad.

\section{PISTAS PARA IDENTIFICAR LA JUSTICIA DESDE LAS MUJERES EN LA AMAZONÍA}

En este contexto de destrucción y surgimiento de nuevas formas de violencia contra las mujeres por la operatividad de las industrias extractivas bajo el modelo de capitalismo por despojo, las mujeres indígenas pueden encontrarse también ante nuevas formas de hacer justicia y re-existir. Este postulado está basado en la interculturalidad crítica, entendida como una pedagogía de-colonial que se construye desde las personas oprimidas e incita a ser, sentir, existir, hacer, pensar, mirar, escuchar y saber de otro modo. ${ }^{45}$ Walsh, quien realiza esta propuesta teórica, dice: "la interculturalidad crítica no es un proceso o proyecto étnico, ni tampoco un proyecto de la diferencia

44. Wilfredo Ardito Vega, La promoción del acceso a la justicia en las zonas rurales (Lima: Poder Judicial, Oficina Nacional de Justicia de Paz y Justicia Indígena / Pontifica Universidad Católica del Perú, 2010), 17-46.

45. Catherine Walsh, "Interculturalidad crítica y educación intercultural", en Construyendo interculturalidad crítica, ed. Jorge Viaña Uzieda, Luis Tapia Mealla y Catherine E. Walsh (La Paz: Convenio Andrés Bello / Instituto Interamericano de Integración, 2010). 
en sí, más bien, como argumenta Adolfo Albán, es un proyecto que apunta a la reexistencia y a la vida misma, hacia un imaginario 'otro' y una agencia 'otra' de convivencia -de vivir 'con'- y de sociedad" ${ }^{46}$ Esta teoría es fundamental para pensar la violencia contra las mujeres indígenas no solo como una denuncia, sino con la esperanza y la apertura de ahondar en las resistencias expresadas por las mujeres indígenas en su búsqueda de justicia. Con ello se postula que detrás de todo grupo oprimido hay una voz de insurgencia.

Estas formas de justicia pueden reconocerse desde el pluralismo jurídico emancipador. Antonio Wolkmer propone un pluralismo jurídico que se dinamice desde la gente y abra el campo de reconocimiento de nuevas formas de regulación de conflictos. Ello puede suscitarse con las mujeres indígenas de la Amazonía, que, en un contexto de impunidad, destrucción e indiferencia, busquen formas de dar respuesta a la violencia que particularmente las afecta. Estas formas de justicia trascienden el modelo normativo de matriz eurocéntrica de control y regulación, pues es precisamente este modelo el que ha generado una crisis al no responder adecuadamente frente a los casos que se suscitan en su contra: la violencia contra las mujeres en contextos extractivos no es atendida y la violencia institucional contra las mujeres perenniza la impunidad frente a sus casos, revictimizando y dando un mensaje de indiferencia.

Por otro lado, la justicia indígena no siempre atiende los casos de violencia contra las mujeres. ${ }^{47}$ Así como la violencia institucional se expresa mediante las barreras de acceso a justicia ordinaria, existen barreras para el acceso a la justicia indígena. Aura Cumes, en el caso guatemalteco, resalta la dicotomía entre el sistema de justicia indígena y ordinaria: "Frente al derecho estatal, las mujeres indígenas se enfrentan a un sistema racista, etnocéntrico y patriarcal, y ante el derecho maya, tienen una posición de subalternidad por ser mujeres". ${ }^{48}$ Es en este escenario en los que pueden surgir nuevas formas de justicia. Para Wolkmer la emergencia es una condición necesaria para que se desarrollen nuevas teorías y proyectos de liberación asentados en prácticas comunitarias. ${ }^{49}$ La propuesta del pluralismo jurídico emancipador parte de la

46. Ibíd., 48 .

47. Ver Rachel Sieder y María Teresa Sierra, "Acceso a la justicia para las mujeres indígenas en América Latina" (Chr. Michelsen Institute en Bergen, 2010); Aura Cumes, "Mujeres indígenas, poder y justicia: de guardianas a autoridades en la construcción de culturas y cosmovisiones", en Mujeres indígenas y justicia ancestral, ed. Miriam Lang y Anna Barrera Vivero (Quito: UNIFEM - Región Andina, 2009); Rocío Franco Valdivia y María Gonzales Luna, Las mujeres en la justicia comunitaria: víctimas, sujetos y actores, Serie Justicia Comunitaria en los Andes: Perú y Ecuador, vol. 3 (Lima: Instituto de Defensa Legal, 2009).

48. Cumes, ibíd., 33.

49. Antônio Carlos Wolkmer, Teoría crítica del derecho desde América Latina, trad. Alejandro Rosillo Martínez (Ciudad de México: Akal, 2017), 201-2. 
identificación de otras formas de justicia en procesos de prácticas sociales insurgentes y nuevos sujetos sociales que pasan a emanciparse..$^{50}$

Las mujeres indígenas se encuentran marginadas en medio de la justicia ordinaria e indígena y, desde esa condición de adversidad, pueden desarrollar alternativas de cambio. Aída Hernández, a partir del estudio de las mujeres zapatistas de Chiapas, resalta las voces críticas de las mujeres frente a la resolución de casos de violencia en su contra por la justicia indígena. ${ }^{51}$ Andrea Pequeño menciona el reclamo de las mujeres indígenas por el reconocimiento de su cultura incluyendo su propia justicia, pero a la par el reclamo a la justicia indígena cuando las excluye, silencia y menoscaba al interior de las órdenes y prácticas de sus comunidades. ${ }^{52}$ En este marco, Pequeño encuentra entre las estrategias de resistencia y/o combate a la violencia que realizaron las mujeres indígenas: los discursos de cara a la violencia al interior de sus familias, comunidades y organizaciones, así como el Estado. ${ }^{53}$ Ello denota una valoración a la voz de las mujeres como forma de defensa.

Con los estudios de la paz y el conflicto se puede ahondar en la transición de víctima a sobreviviente; la sobreviviente busca salir de la situación de crisis y conflicto, desarrollando su agencia. ${ }^{54}$ En ese sentido, las mujeres sobrevivientes de hechos de violencia y aquellas que acompañan y apoyan estos procesos transforman conflictos en oportunidades de repensar la justicia indígena. Irene Comins refiere que las situaciones adversas de conflicto despiertan una resiliencia que fomenta prácticas de cuidado, la praxis del cuidar tiene un potencial poiético bidireccional, de preocupación por el entorno que también satisface a uno mismo: "La persona que cuida se transforma, resignifica, se rehace, a través del ejercicio del cuidar". ${ }^{55}$ En estas situaciones adversas - como cuando un hecho de violencia queda impune en la comunidad-el cuidado es una constante. Las reacciones que identifica Comins son: resistir y movilizarse en nombre de los vínculos, rehacer las condiciones de humanidad, tejer la vida colectiva. $^{56}$

50. Ibíd., 211.

51. R. Aída Hernández Castillo, "El Derecho positivo y la costumbre jurídica: las mujeres indígenas de Chiapas y sus luchas por el acceso a justicia", en Violencia contra las mujeres en contextos urbanos y rurales, ed. Marta Torres Falcón, 1a. ed. (Colegio de México, 2004), 337, doi:10.2307/j.ctv513792.14.

52. Andrea Pequeño Bueno, "Vivir violencia, cruzar los límites", en Participación y políticas de mujeres indigenas en América Latina (Quito: FLACSO Ecuador, 2009), 151.

53. Ibíd.

54. Irene Comins-Mingol, "De víctimas a sobrevivientes: la fuerza poiética y resiliente del cuidar", Convergencia 22, n. 67 (abril de 2015): 39, http://www.scielo.org.mx/scielo.php?script=sci_abstract\&pid=S1405$14352015000100002 \& \operatorname{lng}=\mathrm{es} \&$ nrm $=$ iso\&tlng=es.

55. Ibíd., 41.

56. Ibíd., 45. 
Las nuevas formas de justicia a las que se hacen referencia son parte de la introducción a una propuesta teórica basada en el pluralismo jurídico emancipador, que busca encontrar el surgimiento del derecho y justicia en las respuestas frente a los casos de violencia en contra de las mujeres indígenas de la Amazonía, en un contexto de extractivismo y de precarización de la vida. Existen multiplicidad de sistemas normativos que coexisten en el mundo rural: ¿por qué no se podría tener indicios de otras formas de ejercer justicia dentro de las comunidades?

\section{CONCLUSIONES}

El contexto extractivo en la Amazonía ha generado la precarización de la vida, lo que ha desencadenado nuevas formas de violencia contra las mujeres. Estas formas de violencia se expresan tanto en la trata con fines de explotación sexual, incremento de la violencia física, sexual, psicológica contra las mujeres como también en la violencia relacionada a la limitación de la soberanía alimentaria, la contaminación de los ríos y recursos que altera la dinámica comunitaria. Estas nuevas formas de violencia o violencia intensificada se desatan contra todo lo feminizado: el cuerpo de las mujeres, la naturaleza, la cultura, y afectan tanto los territorios directamente afectados por las actividades extractivas como los territorios aledaños. Es importante que el derecho preste atención a estas nuevas formas de violencia que demandan actualizar e implementar procesos de protección y sanción en estas zonas.

Frente a estas nuevas formas de violencia en contextos extractivos y de precarización, las salidas son limitadas, dada la violencia institucional contra las mujeres. Las barreras de acceso a justicia y la legislación indiferente al contexto cultural de los pueblos indígenas y zonas rurales no responden a políticas adecuadas interculturalmente. En ese mismo sentido, pese a que la justicia indígena es un importante espacio de organización y resistencia dentro de las comunidades, no siempre sanciona los casos de violencia contra las mujeres.

Este escenario de crisis puede dar origen a nuevas prácticas sociales que regulen conflictos. Las mujeres indígenas pueden ser los nuevos sujetos sociales que con su agencia desarrollen prácticas de re-existir. Para ello, se debe entender a las mujeres no como víctimas, sino como sobrevivientes, sujetos capaces de desarrollar acciones de justicia en respuesta a la impunidad y violencias. 


\section{BIBLIOGRAFÍA}

Ardito Vega, Wilfredo. La promoción del acceso a la justicia en las zonas rurales. Lima: Poder Judicial, Oficina Nacional de Justicia de Paz y Justicia Indígena / Pontifica Universidad Católica del Perú, 2010.

Barclayro, Frederica. “¿Qué ha significado el petróleo en la configuración de Loreto como una región?, en Foro 40 años de explotación petrolera en Loreto. Lecciones aprendidas". SPDA Actualidad Ambiental, 23 de noviembre de 2011. http://www.actualidadambiental. pe/wp-content/uploads/2011/11/Qu\%C3\%A9-ha-significado-el-petr\%C3\%B3leo-en-laconsolidaci $\% \mathrm{C} 3 \% \mathrm{~B} 3 n$-de-Loreto-como-una-regi\%C3\%B3n-Frederica-Barclay.pdf.

Bebbington, Denise, Ricardo Verdum, César Gamboa y Anthony Bebbington. Evaluación y alcance de la industria extractiva y la infraestructura en relación con la deforestación: Amazonía. Lima: Derecho, Ambiente y Recursos Naturales (DAR), 2019.

Bodelón, Encarna. "Violencia institucional y violencia de género". Anales de la Cátedra Francisco Suárez. Revista de Filosofía Jurídica y Política 48 (2014). http://revistaseug.ugr.es/ index.php/acfs/article/view/2783.

Bodelón González, Encarna. "Feminismo y Derecho: Mujeres que van más allá de lo jurídico". En Género y dominación: críticas feministas del derecho y el poder, editado por Gemma Nicolás Lazo, Encarna Bodelón González, Roberto Bergalli e Iñaki Rivera, 1a. ed. Huellas. Desafío(s) 7. Rubí, Barcelona: Anthropos, 2009.

Castillo, R. Aída Hernández. "El Derecho postivo y la costumbre jurídica: Las mujeres indígenas de Chiapas y sus luchas por el acceso a justicia”. En Violencia contra las mujeres en contextos urbanos y rurales, editado por Marta Torres Falcón, 1a. ed., 335-78. Colegio de México, 2004. doi:10.2307/j.ctv513792.14.

Comins-Mingol, Irene. "De víctimas a sobrevivientes: la fuerza poiética y resiliente del cuidar". Convergencia 22, n. ${ }^{\circ} 67$ (abril de 2015): 35-54. http://www.scielo.org.mx/scielo. php?script=sci_abstract\&pid=S1405-14352015000100002\&lng=es\&nrm=iso\&tlng=es.

Cumes, Aura. "Mujeres indígenas, poder y justicia: de guardianas a autoridades en la construcción de culturas y cosmovisiones". En Mujeres indígenas y justicia ancestral, editado por Miriam Lang y Anna Barrera Vivero. Quito: UNIFEM - Región Andina, 2009.

Franco Valdivia, Rocío, y María Gonzales Luna. Las mujeres en la justicia comunitaria: víctimas, sujetos y actores. Serie Justicia Comunitaria en los Andes: Perú y Ecuador. Vol. 3. Lima: Instituto de Defensa Legal, 2009.

Gavaldá, Marc. Gas amazónico: los pueblos indígenas frente al avance de las fronteras extractivas en Perú. 1a. ed. Akadēmeia, Ecología humana 142. Barcelona: Icaria, 2013.

Gutiérrez, Raquel, y Verónica Gagó. "Prólogo". En Las nuevas formas de la guerra y el cuerpo de las mujeres. Buenos Aires: Tinta Limón, 2013.

Gutiérrez, Raquel, y Dawn Paley. "La transformación sustancial de la guerra y la violencia contra las mujeres en México". Deportate, esuli, profughe DEP, Rivista telemativa di studi sulla memoria femminile 30 (2016): 12. https://www.unive.it/media/allegato/dep/n302016/01_Aguillar-Dawn-Palley.pdf. 
La Rosa Calle, Javier. “Acceso a la justicia: elementos para incorporar un enfoque integral de política pública”. En Acceso la justicia en el mundo rural, editado por Javier La Rosa Calle. Lima: Instituto de Defensa Legal, 2007.

Lang, Miriam, Margarita Aguinaga, Dunia Mokrani y Alejandra Santillana. "Pensar desde el feminismo: Críticas y alternativas al desarrollo". En Más allá del desarrollo, editado por Grupo Permanente de Trabajo sobre Alternativas al Desarrollo. Quito: Fundación Rosa Luxemburg / Abya-Yala, 2011.

Lapierre Robles, Michel, y Aguasantas Macías Marín. Extractivismo, (neo)colonialismo y crimen organizado en el norte de Esmeraldas. Quito: Abya-Yala / Pontificia Universidad Católica del Ecuador / Instituto de Estudios Ecologistas del Tercer Mundo, 2018.

Mies, María, y Vandana Shiva. Ecofeminismo: teoría, crítica y perspectivas. Barcelona: Icaria, 2014.

Mujica, Jaris. Elementos comparados del impacto de la trata de personas en la salud de víctimas adolescentes en el contexto de la minería ilegal de oro en Madre de Dios. Lima: OMSOPS / Anesvad / PROMSEX Centro de Promoción y Defensa de los Derechos Sexuales y Reproductivos, 2014.

Organización de Estados Americanos. Audiencia sobre denuncias de violaciones de derechos humanos de las mujeres en el contexto de actividades extractivas en Perú. 159 Período de Sesiones, Panamá, 2 de diciembre de 2016.

- Convención Interamericana para Prevenir, Sancionar y Erradicar la Violencia contra la Mujer (Convención de Belém do Pará), 9 de junio de 1994.

Organización de los Estados Americanos, y Comisión Interamericana de Derechos Humanos. "Las mujeres indígenas y sus derechos humanos en las Américas". Organización de los Estados Americanos, 2017.

Organización de Naciones Unidas, Comité para la eliminación de todas las formas de discriminación contra la mujer. Recomendación general $n .^{\circ} 30$, sobre las mujeres en la prevención de conflictos y en situaciones de conflicto y posteriores a conflictos. $56^{\circ}$ Período de sesiones, 1 de noviembre de 2013.

Pequeño Bueno, Andrea. "Vivir violencia, cruzar los límites". En Participación y políticas de mujeres indigenas en América Latina. Quito: FLACSO Ecuador, 2009.

Segato, Rita. La violencia contra las mujeres es sobre las mujeres contra la vida. Entrevistado por Nadia Fink. Fundación Rosa Luxemburgo, 7 de agosto de 2018. http://www.biodiversidadla.org/Documentos/La_violencia_contra_las_mujeres_es_sobre_las_mujeres_contra_la_vida._Dialogo_con_Rita_Segato.

Segato, Rita Laura. "La argamasa jeráquica: Violencia moral, reproducción del mundo y eficacia simbólica del derecho". En Las estructuras elementales de la violencia: Ensayos sobre género entre la antropología, el psicoanálisis y los derechos humanos, 1a. ed. Derechos humanos. Viejos problemas, nuevas miradas. Buenos Aires: Universidad Nacional de Quilmes, Prometeo 3010, 2003.

- Las nuevas formas de la guerra y el cuerpo de las mujeres. Buenos Aires: Tinta Limón, 2013. 
Sieder, Rachel, y María Teresa Sierra. "Acceso a la justicia para las mujeres indígenas en América Latina". Chr. Michelsen Institute en Bergen, 2010. https://www.cmi.no/publications/ file/3941-acceso-a-la-justicia-para-las-mujeres-indigenas-en.pdf.

Sierra Praeli, Yvette. "¿Qué ha pasado en 40 años de actividad petrolera en la Amazonía peruana?". MONGOBAY: Periodismo ambiental independiente, 23 de junio de 2017. https:// es.mongabay.com/2017/06/ha-pasado-40-anos-actividad-petrolera-la-amazonia-peruana/.

Silva Santisteban, Rocío. Mujeres y conflictos ecoterritoriales: impactos, estrategias, resistencias. Lima: Centro de la Mujer Peruana Flora Tristán / DEMUS Estudio para la Defensa de los Derechos de la Mujer, Coordinadora Nacional de Derechos Humanos / AIETI Asociación de Investigación y Especialización sobre Temas Iberoamericanos / Entre pueblos, 2017.

Ulloa, Astrid. "Feminismos territoriales en América Latina: defensas de la vida frente a los extractivismos NÓMADAS 45 | octubre de 2016 - Universidad Central - Colombia". NÓMADAS, n. ${ }^{\circ} 45$ (octubre de 2016): 123-39. http://nomadas.ucentral.edu.co/index.php/component $/$ content/article?id $=887$.

Walsh, Catherine. "Interculturalidad crítica y educación intercultural". En Construyendo interculturalidad crítica, editado por Jorge Viaña Uzieda, Luis Tapia Mealla y Catherine E. Walsh. La Paz: Convenio Andrés Bello / Instituto Interamericano de Integración, 2010.

Wolkmer, Antônio Carlos. Teoría crítica del derecho desde América Latina. Traducido por Alejandro Rosillo Martínez. Akal-Inter Pares. Ciudad de México: Akal, 2017. 